\title{
Gregor Perko
}

Filozofska fakulteta Univerze v Ljubljani

gregor.perko@ff.uni-lj.si

\section{ZDEŇKA SCHEJBALOVÁ: L'ÉVOLUTION DES FONCTIONS DE L'ARTICLE PARTITIF EN FRANÇAIS}

Schejbalová, Zdeňka (2003): L'évolution des fonctions de l'article partitif en français. Brno: Masarykova univerzita, Pedagogická fakulta. ISBN 80-210-3229-4, broché, 50 kč

Le livre, comme son titre l'indique, apporte une étude diachronique de l'évolution de l'emploi de l'article partitif en français. Il est inutile de dire que l'emploi de cet article pose de sérieux problèmes aux apprenants étrangers. En principe, sa valeur sémantico-syntaxique semble assez nette. L'article partitif est en distribution complémentaire avec l'article indéfini (un, une, des) et, de même que l'article indéfini pluriel des, il est porteur d'une double indétermination : quant à l'identité et quant à la quantité. Le fonctionnement de l'article partitif dans le français courant moderne s'avère être, tant s'en faut, moins simple, englobant des emplois dans la sphère du concret, de l'abstrait, du figuré, voire de l'affectif.

Après trois chapitres introducteurs, l'ouvrage s'organise autour de l'axe chronologique, couvrant la quasi-totalité de l'histoire de la langue française, allant du $\mathrm{IX}^{\mathrm{e}}$ au $\mathrm{XX}^{\mathrm{e}}$ siècles. L'auteur commence par rattacher la sémantique de l'article partitif à la notion de partitivité. Dans les langues indo-européennes, cette notion, d'ordre sémantique, peut se réaliser de deux façons morphosyntaxiques différentes : soit sous une forme synthétique, au moyen du génitif partitif marquant à la fois la dépendance du verbe recteur et la partitivité, soit sous une forme analytique, au moyen de l'ablatif précédé de la préposition de. La forme synthétique, considérée par les linguistes comme la plus ancienne, a disparu dans la plupart des langues indo-eurpéennes modernes et a été " remplacé » par l'accusatif ou le complément verbal direct, qui ne comportent plus aucune marque morphosyntaxique de la partitivité. Le slovène semble bien refléter cettte "évolution ». Au côté de la construction « non marquée » Kupil sem krub ( fr. [Je] ai acheté pain accusatif), subsite la construction avec le génitif, considérée aujourd'hui comme archaïque Kupil sem kruha ( fr. [Je] ai acheté paingénitif).

Les langues romanes ont tout naturellement « hérité » de la forme analytique de+ablatif du latin vulgaire, celle-ci étant cependant progressivement abandonnée par la plupart des langues romanes, à l'exception de l'italien et du français, qui sont les seuls à avoir développé l'article partitif, mais aussi du provençal et des dialectes centraux du sarde qui « continuent » à employer seule la préposition/particule de seule. L'origine de l'article partitif (verbe $+d e+$ ablatif) permet 
de mieux comprendre la tendance encore actuelle de l'article partitif en français d'apparaître essentiellement en position de complément de verbe et rarement en position de sujet.

La perspective diachronique, doublée d'une approche philologique, permet à l'auteur de remettre en quetion l'hypothèse de G. Guillaume, avancée dans sa célèbre étude Le problème de l'article et sa solution dans la langue française, selon laquelle l'évolution de l'article partitif connaîtrait trois étapes : $0>d e>d u$. Selon Schejbalová, cette évolution adopterait plutôt le parcours $0 / d e>d e / d u>d u$.

Jusqu'au XII ${ }^{e}$ siècle, l'emploi de l'article partitif n'est pas attesté, la production écrite se pliant sans doute à la "norme » latine. Les XII et XIII ${ }^{e}$ siècles connaissent la concurrence $0 / d e / d u$. On relève déjà des occurrences de l'article partitif précédant les noms abstraits et même comptables. Jusqu'au XVI ${ }^{\mathrm{e}}$ siècle l'emploi du partitif se régularise, quoique le partitif soit encore fortement concurrencé par l'article zéro. On peut dire que le système actuel de l'article français, dont fait partie le partitif, prend définitivement forme au XVII ${ }^{e}$ siècle. Les siècles ultérieurs voient la consolidation des emplois prototypiques ainsi que le développement d'emplois figurés, affectifs ou ludiques.

L'ouvrage, qui se base sur un corpus de plus de 2000 occurrences tirées de plus de 50 oeuvres, essentiellement littéraires, apportent des renseignements précieux tant à celui qui s'intéresse à la linguistique diachronique qu'à celui qui étudie le fonctionnement du système morphosyntaxique en synchronie. 